\title{
ASSOCIATION OF THE MTHFR C677T (rs1801133) POLYMORPHISM WITH IDIOPATHIC MALE INFERTILITY IN A LOCAL PAKISTANI POPULATION
}

\author{
Irfan $\mathrm{M}^{1, *}$, Ismail M${ }^{2}$, Azhar Beg $\mathrm{M}^{1}$, Shabbir A², Rashid Kayani A ${ }^{1}$, Kaukab Raja $\mathrm{G}^{4}$
}

*Corresponding Author: Muhammad Irfan, M.Phil., Department of Zoology, Pir Mehr Ali Shah Arid Agriculture University Rawalpindi, Pakistan. Tel: +92-344-551-8382. Email: muhammadirfan11@gmail.com

\begin{abstract}
The present study determined an association between idiopathic sperm disorders in a local Pakistani infertile male population and the MTHFR C677T polymorphism. After ruling out non genetic factors, a total of 437 idiopathic infertile men including 57 azoospermic, 66 oligospermic, 44 asthenozoospermic, 29 teratozoospermic, 20 oligoasthenospermic and 221 infertile normospermic men were recruited. Furthermore, 218 normospermic fertile men, who had two children (or more) were included as controls. The polymerase chain reaction-restriction fragment length polymorphism (PCR-RFLP) technique was used to determine MTHFR C677T (rs1801133) polymorphism. A significant association of the minor MTHFR $677 \mathrm{~T}$ allele with male infertility was observed $(p$ $<0.05)$. In addition, men with MTHFR $677 \mathrm{CT}$ and TT genotypes were at a greater risk [odds ratio (OR): $1.81,95 \%$ confidence interval $(95 \% \mathrm{CI}): 1.17-2.80$, $p=0.008$ and OR: $9.24,95 \%$ CI: $1.20-70.92, p=$ 0.032 , respectively] of infertility. All the subgroups of male infertility (azoospermic, oligospermic, as-
\end{abstract}

\footnotetext{
${ }^{1}$ Department of Zoology, Pir Mehr Ali Shah-Arid Agriculture University, Rawalpindi, Pakistan

${ }^{2}$ Institute of Biomedical and Genetic Engineering (IBGE), Islamabad, Pakistan

${ }^{3}$ Biosciences, Commission on Science and Technology for Sustainable Development in the South (COMSAT), Institute of Information Technology, Islamabad, Pakistan

${ }^{4}$ Department of Biochemistry, Pir Mehr Ali Shah-Arid Agriculture University, Rawalpindi, Pakistan
}

thenospermic, oligoasthenoteratospermic (OAT) and normospermic infertile) had significantly $(p<0.05)$ higher frequencies of $\mathrm{CT}$ and TT genotypes when compared to fertile men. The combined genotypes $(\mathrm{CT}+\mathrm{TT})$ were also found significantly (OR: 2.01, 95\% CI: 1.31-3.08, $p<0.001)$ associated with male infertility. The results suggest that the polymorphism might be a factor of male infertility in the Pakistani population.

Keywords: Male Infertility; MTHFR; Polymorphism; Reproduction; Sperm disorders.

\section{INTRODUCTION}

Infertility is a state of failure to conceive in a year of unprotected intercourse with the same partner [1]. The male factors are responsible of infertility in 20.0 to $25.0 \%$ of couples [2]. In men, the main causes of infertility are oligospermia, asthenospermia, teratozoospermia and azoospermia $[3,4]$. Like other nutritional factors, availability of bioactive folate has recently been found to be related to male fertility [5].

Folates are an inter-convertible group of enzymes that metabolize amino acid, synthesize and methylate deoxy-ribonuleotides (dNTPs) [6,7]. Methyltetrahydrofolate (MethylTHF) is the biologically active form of folate in plasma. An important enzyme, methylenetetrahydrofolate reductase (MTHFR), reduces 5,10 methylenetetrahydrofolate into 5, MethylTHF and during this reduction, a homocysteine is converted into methionine by attaining a methyl group from methylenetetrahydrofolate [8]. 
Methionine is converted to S-adenosyl methionine that acts as a 'methyl' donor for DNA methylation. The 5,10 methylenetetrahydrofolate converts uracil into thymine for DNA synthesis [9]. Therefore, deficiency in folate intake or polymorphism(s) in the enzymes of the folate pathway may result in aberrant DNA synthesis and methylation, especially in rapidly dividing cells such as bone marrow and spermatogonia [10].

The MTHFR gene is composed of 11 exons and is located on the short arm of chromosome 1 at $1 \mathrm{p} 36.22$ $[8,11]$. Being an important enzyme of folate metabolism, the MTHFR gene product has been studied and found in a total of 65 polymorphisms. The C677T (rs1801133) polymorphism in the MTHFR gene reduces the enzyme activity by $35.0 \%$ [11] resulting in impairment of nucleic acid metabolic pathways. However, the condition can be managed by folic acid supplementation for determining the frequency of the MTHFR C677T mutation is a prerequisite.

The results of many of the molecular epidemiological studies on the association of the MTHFR C677T polymorphism and male infertility remain controversial due to small sample size, ambiguously defining infertility and confounding factors including ethnicity. In the present study, we determined an association between idiopathic sperm disorders and the MTHFR 677 CT polymorphism.

\section{MATERIALS AND METHODS}

Sample Population. Initially, 1234 men with primary infertility (the couples never conceived) were recruited from various diagnostic setups, hakeems (Muslim physicians) and private clinics in two cities of Rawalpindi and Islamabad, Pakistan, for a period of 2 years (2011 and 2012). A cohort of 348 proven fathers (had at least two children), residents of the same area, was also taken as a control group.

Inclusion and Exclusion Criteria. After approval and permission of the concerned authorities, infertile men fulfilling the World Health Organization (WHO) criteria of failure to conceive during 1 year of unprotected intercourse with the same partner were recruited [1]. Only those men, whose partners were already screened and had normal reproductive functions, were included. The men with other physiological disorders, congenital and psychological disorders including diabetes, allergies, timing of the onset of puberty, cryptorchidism, testicular torsion, ectopic testis, single testis and testicular damage at birth and varicocele were excluded. The men with mental disorders, i.e., depression [12], anxiety [13] and stress [14], screened by using standard scales, respectively, were also excluded. The past and present infections (mumps, high fever, tuberculosis, lung infections, reproductive tract infections and antisperm antibodies) and testicular or abdominal injury/surgery or vasectomy and Y-chromosome micro-deletions, were also excluded. Moreover, men (both fertile and infertile) with hormonal disorders [tri-iodothyronine $\left(\mathrm{T}_{3}\right)$, tetra-iodothyronine $\left(\mathrm{T}_{4}\right)$, thyroid stimulating hormone (TSH), follicle stimulating hormone (FSH), luteinizing hormone ( $\mathrm{LH})$, testosterone $(\mathrm{T})$ and prolactin], and suspected lifestyle factors [smoking $>5$ cigarette $(\sim 5 \mathrm{gm}$ tobacco)/day; tea/coffee/soft drinks (one or all) $>3$ times/day or $>600 \mathrm{~mL}$ (three cups)/day; wearing tight clothes $>3$ days a week and $>12$ hours/day for at least 1 year] were also excluded from the study.

Consequently, a total 437 idiopathic infertile men including 57 azoospermic, 66 oligospermic, 44 asthenozoospermic, 29 teratozoospermic, 20 oligoasthenospermic and 221 infertile normospermic men were recruited, after ruling out nongenetic factors. Moreover, 218 normospermic fertile men who had two children (or more), were included as controls. The samples and controls screened for MTHFR C677T belonged to the Punjabi ethnic group as selfdefined by subjects.

Semen Sampling. The semen samples were initially collected for analysis of a suspected fertility problem in men. The semen samples were also obtained from fertile men (control group). Each individual gave semen samples twice by masturbation after 3-5 days of abstinence, their written consent was obtained for the sample to be used further in molecular research.

Blood Sampling. At least $10 \mathrm{~mL}$ of a single blood sample was collected from the antecubital vein of each subject and stored in vacutainers with EDTA as anticoagulant. Blood samples were immediately transported to the Genetics Laboratory, Institute of Biomedical Sciences and Genetic Engineering (IBGE), Islamabad, Pakistan, where the plasma was separated from all samples by cen-trifugation and stored at $-25{ }^{\circ} \mathrm{C}$ until further analysis. The cellular portion was used for DNA extraction for genetic analysis. 


\section{SAMPLE ANALYSES}

Semen Analysis. Semen parameters (semen volume, sperm concentration, sperm motility, sperm morphology, liquefaction time, $\mathrm{pH}$ and colour) were determined in a private laboratory by an expert using standard methods. The semen samples were categorized into one of the semen group defined by WHO [1]. (Table 3.1). The volume was measured using a graduated glass pipette. The sperm concentration was counted using a sperm counting chamber (Jiansu Sanwe Medical Science and Technology Co. Ltd., Xuzhou, China). The concentration, motility and morphology of sperm cells were observed using a binocular microscope at x100 magnification (Olympus Optical Co. Ltd., Tokyo, Japan). The semen pH was determined with a digital $\mathrm{pH}$ meter.

Genetic Analysis. Total genomic DNA was extracted from blood samples using a standard phenol/ chloroform extraction method.

MTHFR C677T Genotyping. The MTHFR C677T polymorphism was analyzed by polymerase chain reaction (PCR) of the genomic DNA with primers 5'-ACC CAC AGA AAA TAC CCA G-3' (forward) and 5'-TGC CCC ATT ATT TA-3' (reverse) (Alpha DNA, Montreal, Quebec, Canada) with an initial step consisting of denaturation for $4 \mathrm{~min}$. at 94 ${ }^{\circ} \mathrm{C}$, annealing for 45 seconds at $60^{\circ} \mathrm{C}$, extension for 45 seconds at $72{ }^{\circ} \mathrm{C}$, followed by 35 cycles of denaturation at $94{ }^{\circ} \mathrm{C}$ for 45 seconds, annealing at $60{ }^{\circ} \mathrm{C}$ for 45 seconds, extension at $72^{\circ} \mathrm{C}$ for 45 seconds, and a final extension at $72{ }^{\circ} \mathrm{C}$ for $10 \mathrm{~min}$. The amplified PCR products were digested with Hinfl restriction endonuclease (Fermentas Life Sciences, Burlington, ON, Canada) as the C677T polymorphism creates a restriction site for it.

Gel Electrophoresis. The digested product was elec-trophoresed on a 3.0\% agarose gel with ethidium bromide staining which was then visualized through ultraviolet transillumination. The normal allele with cytosine at position 677 (C677) formed an undigested fragment of $198 \mathrm{bp}$, while the mutant allele with thymine in position 677 (T677) formed fragments of 175 and $23 \mathrm{bp}$.

Statistical Analysis. The allele frequency of the MTHFR $677 \mathrm{C}>\mathrm{T}$ polymorphism was determined by counting alleles through electrophoresis gel analysis. The $\chi^{2}$ analysis was used to determine the HardyWeinberg equilibrium of the alleles in the population.

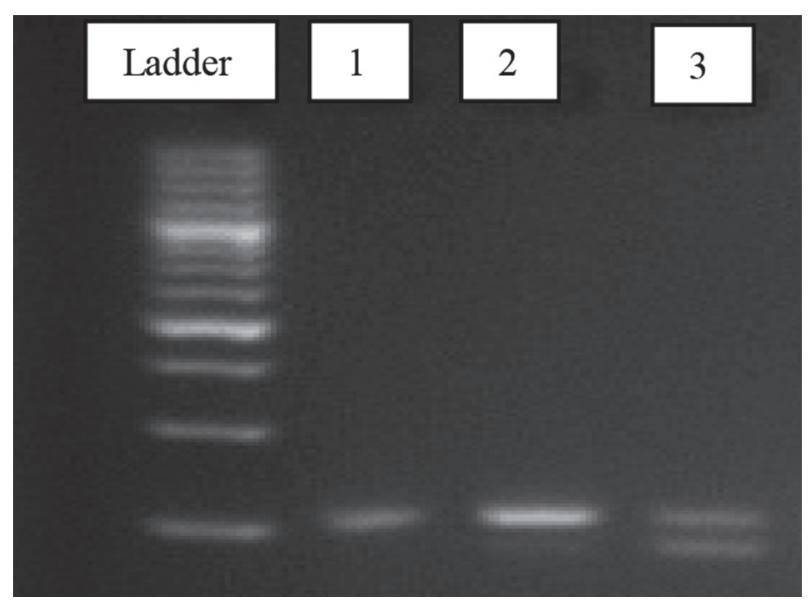

Figure 1. DNA electrophoresis of the MTHFR C677T mutation on $3.0 \%$ agarose gel. 1: undigested; homozygous normal allele (CC) 198 bp; 2 and 3: heterozygous allele (CT) 175 and 23 bp.

The association of the MTHFR $677 \mathrm{C}>\mathrm{T}$ polymorphism with subgroups of male infertility was determined by logistic regression analysis adjusting the effects of age, body mass index (BMI), occupation, working hours, and working shift. A $p$ value of $<0.05$ was considered statistically significant. All statistical analyses were performed using the International Business Machines (IBM) Statistical Package for the Social Sciences (SPSS) v20.0 for Windows (IBM SPSS; http:// www.ibm.com).

\section{RESULTS}

Genotype distribution of the MTHFR $677 \mathrm{C}>\mathrm{T}$ polymorphism of the 655 subjects analyzed: 472 $(72.06 \%)$ subjects were homozygous for the $\mathrm{C}$ allele (CC), 166 (25.34\%) subjects were heterozygous (CT), and $17(2.60 \%)$ subjects were homozygous for the $\mathrm{T}$ allele (TT). The minor allele (T) frequency of the MTHFR $677 \mathrm{C}>\mathrm{T}$ polymorphism was 0.153 , and the genotype distributions did not deviate from the HardyWeinberg equilibrium $(p>0.05)$ (Tables 1 and 2).

Prevalence and Odds Ratios of Male Infertility According to Carriers of the Minor Allele of the MTHFR C677T Polymorphism. a) Allele frequencies. The relative MTHFR 677 allele and genotype frequencies of the individual risk factors of the male infertility in the total populations are summarized in Tables 1 and 2. Results show that the MTHFR $\mathrm{C}>\mathrm{T}$ polymorphism increases the odds ratio $(\mathrm{OR})[2.12,95 \%$ confidence interval $(95 \% \mathrm{CI})$ : $1.38-3.92]$ of male infertility significantly $(p<0.05)$. 
Table 1. Allele frequencies of the MTHFR C677T mutation in the studied subjects.

\begin{tabular}{|c|c|c|c|c|c|c|c|}
\hline Patient Group & Allele & $\begin{array}{c}\text { Infertile Men } \\
n(\%)\end{array}$ & $\begin{array}{c}\text { Fertile Men } \\
\quad n(\%)\end{array}$ & OR $(95 \% \mathrm{CI})$ & $p$ Value & AOR $(95 \% \mathrm{CI})$ & $p$ Value \\
\hline Azoospermic & $\begin{array}{l}\mathrm{C} \\
\mathrm{T}\end{array}$ & $\begin{array}{l}90(78.95) \\
24(21.05)\end{array}$ & $\begin{array}{c}404(92.66) \\
32(7.34)\end{array}$ & $\begin{array}{c}1 \\
3.37(1.89-5.99)\end{array}$ & 0.000 & $\begin{array}{c}1 \\
2.05(1.14-3.70)\end{array}$ & $0.017^{\mathrm{a}}$ \\
\hline Oligospermic & $\begin{array}{l}\mathrm{C} \\
\mathrm{T}\end{array}$ & $\begin{array}{l}95(71.97) \\
37(28.03)\end{array}$ & $\begin{array}{c}404(92.66) \\
32(7.34)\end{array}$ & $\begin{array}{c}1 \\
4.92(2.91-8.30)\end{array}$ & 0.000 & $\begin{array}{c}1 \\
4.36(2.52-7.53)\end{array}$ & $0.000^{\mathrm{a}}$ \\
\hline Asthenozoospermic & $\begin{array}{l}\mathrm{C} \\
\mathrm{T}\end{array}$ & $\begin{array}{l}64(72.73) \\
24(27.27)\end{array}$ & $\begin{array}{c}404(92.66) \\
32(7.34)\end{array}$ & $\begin{array}{c}1 \\
3.70(2.09-6.52)\end{array}$ & 0.000 & $\begin{array}{c}1 \\
3.70(1.98-6.89)\end{array}$ & $0.001^{\mathrm{a}}$ \\
\hline Teratozoospermic & $\begin{array}{l}\mathrm{C} \\
\mathrm{T}\end{array}$ & $\begin{array}{l}41(70.69) \\
17(29.31)\end{array}$ & $\begin{array}{c}404(92.66) \\
32(7.34)\end{array}$ & $\begin{array}{c}1 \\
5.23(2.68-10.23)\end{array}$ & 0.000 & $\begin{array}{c}1 \\
3.31(1.61-6.82)\end{array}$ & $0.000^{\mathrm{a}}$ \\
\hline OAT & $\begin{array}{l}\mathrm{C} \\
\mathrm{T}\end{array}$ & $\begin{array}{l}29(72.50) \\
11(27.50)\end{array}$ & $\begin{array}{c}404(92.66) \\
32(7.34)\end{array}$ & $\begin{array}{c}1 \\
2.39(1.14-5.03)\end{array}$ & 0.021 & $\begin{array}{c}1 \\
3.33(1.37-8.07)\end{array}$ & $0.008^{\mathrm{a}}$ \\
\hline Infertile normospermic & $\begin{array}{l}\mathrm{C} \\
\mathrm{T}\end{array}$ & $\begin{array}{r}387(87.56) \\
55(12.44)\end{array}$ & $\begin{array}{c}404(92.66) \\
32(7.34)\end{array}$ & $\begin{array}{c}1 \\
0.90(0.61-1.32)\end{array}$ & 0.581 & $\begin{array}{c}1 \\
1.77(1.10-2.85)\end{array}$ & $0.018^{\mathrm{a}}$ \\
\hline Total infertile subjects & $\begin{array}{l}\mathrm{C} \\
\mathrm{T}\end{array}$ & $\begin{array}{l}706(80.78) \\
168(19.22)\end{array}$ & $\begin{array}{c}404(92.66) \\
32(7.34)\end{array}$ & $\begin{array}{c}1 \\
1.50(1.10-2.05)\end{array}$ & 0.012 & $\begin{array}{c}1 \\
2.12(1.38-3.92)\end{array}$ & $0.016^{\mathrm{a}}$ \\
\hline
\end{tabular}

OR: odds ratio; 95\% CI: 95\% confidence interval; AOR: odds ratio adjusted for age, body mass index (BMI), occupation, working shift and hours; C: wild type; T: mutated; OAT: oligoasthenoteratospermic.

a A $p$ value of $<0.05$ was considered to be significant.

Table 2. Genotype frequencies of the MTHFR C677T mutation in the studied subjects.

\begin{tabular}{|c|c|c|c|c|c|c|c|}
\hline Patient Group & Genotype & $\begin{array}{c}\text { Infertile Men } \\
n(\%) \\
\end{array}$ & $\begin{array}{c}\text { Fertile Men } \\
n(\%)\end{array}$ & OR $(95 \% \mathrm{CI})$ & $p$ Value & $\operatorname{AOR}(95 \% \mathrm{CI})$ & $p$ Value \\
\hline \multirow{4}{*}{ Azoospermic } & $\mathrm{CC}$ & $36(63.16)$ & $187(85.78)$ & 1 & & 1 & \\
\hline & $\mathrm{CT}$ & $18(31.58)$ & $30(13.76)$ & $3.12(1.57-6.186)$ & 0.001 & $2.59(1.27-5.30)$ & $0.009^{\mathrm{a}}$ \\
\hline & TT & $3(5.26)$ & $1(0.46)$ & $15.58(1.58-154.06)$ & 0.019 & $13.30(1.23-33.20)$ & $0.033^{\mathrm{a}}$ \\
\hline & $\mathrm{CT}+\mathrm{TT}$ & $21(36.84)$ & $31(14.22)$ & $3.52(1.82-6.80)$ & 0.000 & $2.12(1.09-4.15)$ & $0.027^{\mathrm{a}}$ \\
\hline \multirow{4}{*}{ Oligospermic } & $\mathrm{CC}$ & $34(51.52)$ & $187(85.78)$ & 1 & & 1 & \\
\hline & $\mathrm{CT}$ & $27(40.91)$ & $30(13.76)$ & $4.95(2.62-9.35)$ & 0.000 & $4.33(2.18-8.59)$ & $0.000^{\mathrm{a}}$ \\
\hline & TT & $5(7.58)$ & $1(0.46)$ & $27.50(3.12-242-77)$ & 0.003 & $24.05(2.54-87.34)$ & $0.006^{\mathrm{a}}$ \\
\hline & $\mathrm{CT}+\mathrm{TT}$ & $32(48.48)$ & $31(14.22)$ & $5.58(3.07-10.49)$ & 0.000 & $5.01(2.58-9.71)$ & $0.000^{\mathrm{a}}$ \\
\hline \multirow{4}{*}{ Asthenozoospermic } & $\mathrm{CC}$ & $22(50.00)$ & $187(85.78)$ & 1 & & 1 & \\
\hline & $\mathrm{CT}$ & $20(45.45)$ & $30(13.76)$ & $5.67(2.76-11.62)$ & 0.000 & $5.10(2.32-11.23)$ & $0.000^{\mathrm{a}}$ \\
\hline & TT & $2(4.55)$ & $1(0.46)$ & $17.00(1.48-195.20)$ & 0.023 & $15.93(1.22-97.81)$ & $0.035^{\mathrm{a}}$ \\
\hline & $\mathrm{CT}+\mathrm{TT}$ & $22(50.00)$ & $31(14.22)$ & $6.03(2.99-12.18)$ & 0.000 & $5.03(2.35-10.75)$ & $0.000^{\mathrm{a}}$ \\
\hline \multirow{4}{*}{ Teratozoospermic } & $\mathrm{CC}$ & $15(51.72)$ & $187(85.78)$ & 1 & & 1 & \\
\hline & $\mathrm{CT}$ & $11(37.39)$ & $30(13.76)$ & $4.57(1.92-10.89)$ & 0.000 & $2.90(1.09-7.76)$ & $0.033^{\mathrm{a}}$ \\
\hline & TT & $3(10.34)$ & $1(0.46)$ & $37.40(3.66-381-91)$ & 0.002 & $30.99(2.91-82.3)$ & $0.004^{\mathrm{a}}$ \\
\hline & $\mathrm{CT}+\mathrm{TT}$ & $14(48.28)$ & $31(14.22)$ & $5.63(2.48-12.80)$ & 0.000 & $3.17(1.27-7.91)$ & $0.014^{\mathrm{a}}$ \\
\hline \multirow{4}{*}{ OAT } & $\mathrm{CC}$ & $10(50.00)$ & $187(85.78)$ & 1 & & 1 & \\
\hline & $\mathrm{CT}$ & $9(45.00)$ & $30(13.76)$ & $5.61(2.12-14.94)$ & $\begin{array}{l}0.000 \\
0.044\end{array}$ & $4.10(0.23-13.19)$ & $0.018^{\mathrm{a}}$ \\
\hline & TT & $1(5.00)$ & $1(0.46)$ & $18.70(1.09-321.32)$ & $\begin{array}{l}0.044 \\
0.000\end{array}$ & $14.68(0.61-68.20)$ & 0.098 \\
\hline & $\mathrm{CT}+\mathrm{TT}$ & $10(50.00)$ & $31(14.22)$ & $6.03(2.32-15.68)$ & 0.000 & $4.55(1.46-14.14)$ & $0.009^{\mathrm{a}}$ \\
\hline \multirow{4}{*}{ Infertile normospermic } & $\mathrm{CC}$ & $168(76.02)$ & $187(85.78)$ & 1 & & 1 & \\
\hline & $\mathrm{CT}$ & $51(23.08)$ & $30(13.76)$ & $1.89(1.15-3.12)$ & 0.012 & $1.92(1.12-3.27)$ & $0.017^{\mathrm{a}}$ \\
\hline & TT & $2(0.90)$ & $1(0.46)$ & $2.23(0.20-24.77)$ & 0.515 & $2.48(0.22-28.28)$ & 0.464 \\
\hline & $\mathrm{CT}+\mathrm{TT}$ & $53(23.98)$ & $31(14.22)$ & $1.90(1.17-3.12)$ & 0.010 & $1.95(1.17-3.26)$ & $0.011^{\mathrm{a}}$ \\
\hline \multirow{4}{*}{ Total infertile subjects } & $\mathrm{CC}$ & $285(65.22)$ & $187(85.78)$ & 1 & & 1 & \\
\hline & CT & $136(31.12)$ & $30(13.76)$ & $2.97(1.92-4.60)$ & 0.000 & $1.81(1.17-2.80)$ & $0.008^{\mathrm{a}}$ \\
\hline & TT & $16(3.66)$ & $1(0.46)$ & $10.50(1.38-79.83)$ & 0.023 & $9.24(1.20-70.92)$ & $0.032^{\mathrm{a}}$ \\
\hline & $\mathrm{CT}+\mathrm{TT}$ & $152(34.78)$ & $31(14.22)$ & $3.22(2.10-4.94)$ & 0.000 & $2.01(1.31-3.08)$ & $0.001^{\mathrm{a}}$ \\
\hline
\end{tabular}

OR: odds ratio; 95\% CI: 95\% confidence interval; AOR: odds ratio adjusted for age, body mass index (BMI), occupation, working shift and hours; C: wild type; T: mutated; OAT: oligoasthenoteratospermic.

a A $p$ value of $<0.05$ was considered to be significant. 
In addition, after classifying the men into different infertility groups, allelic frequencies were significantly different between infertile and fertile men. The minor allele ( $\mathrm{T}$ ) frequency was highest in teratospermic men $(0.293 \%)$, followed by oligospermic $(0.280 \%)$, oligoasthenoteratospermic (OAT) $(0.275 \%)$, asthenospermic $(0.273 \%)$, azoospermia $(0.210 \%)$, normospermic infertile $(0.124 \%)$ and fertile men $(0.073 \%)$ (Table 1$)$.

Genotype Frequencies. According to Table 2, men with MTHFR $677 \mathrm{CT}$ and TT genotypes are at a greater risk (OR: 1.81, $95 \% \mathrm{CI}: 1.17-2.80, p=$ 0.008 and OR: $9.24,95 \%$ CI: $1.20-70.92, p=0.032$, respectively) of male infertility. All the subgroups of male infertility (azoospermic, oligospermic, asthenospermic, OAT and normospermic infertile) had significantly $(p<0.05)$ higher frequencies of CT and TT genotype as compared to fertile men. The combined genotypes $(\mathrm{CT}+\mathrm{TT})$ were also found to be significantly associated with male infertility (OR: 2.01, $95 \%$ CI: 1.31-3.08, $p<0.001$ ) (Table 2).

\section{DISCUSSION}

Spermatogenesis is a complex process involving about 2000 genes and various non genetic factors. The gene mutations and disrupted expression may distort maturation of spermatocytes. DNA methylation ensures the regulated expression of genes. However, DNA methylation is done only in the presence of methionine. A homocysteine is converted into methionine by MTHFR through transferring a methyl group from methylenetetrahyfrofolate [8]. Therefore, a mutation in the MTHFR such as C677T may reduce methylation of DNA [11]. In addition, a reduced conversion of homocysteine into methionine may lead to hyper-homocysteinemia, and cause DNA and cell membrane damages, testicular arterial sclerosis, and impairment of spermatogenesis [11,15-17].

However, results of most of the molecular epidemiological studies on association of MTHFR $677 \mathrm{CT}$ polymorphism and male infertility remain controversial. A total of 20 of 41 studies showed association of the polymorphism with male infertility. Out of four [18-21] studies carried out on African populations, only one ([18] showed a significant association of the 677T with infertility in Egyptian men. Furthermore, a study on Moroccan infertile men showed a protective role of the MTHFR 677T polymorphism [22]. Comparatively, more than half the number of Asian studies (14 out of 22) [22-32,36-38,41-42] expressed this association, while only a few Caucasian studies (six out of 15) [44-47,50-51] exhibited it.

Similarly, all the studies [22-28] except one [29] reported a significant association of the $677 \mathrm{~T}$ anomaly with male infertility. Seven studies on Indian populations reported contrasting observations to each other [30-36]. Four Indian studies [30-33] showed a significant association of mutant genotypes with infertility, while three studies [34-36] showed no statistically significant difference of $677 \mathrm{C}>\mathrm{T}$ variants between infertile and fertile males. Dhillon et al. [35] explained the variations in results that their study included a majority of OAT patients in comparison to the majority of azoospermic cases in the study by Singh et al. [29]. Gupta et al. [30] included both azoospermic and OAT individuals, showing a significant association of mutant alleles and genotypes with infertility. It was reported that the three Indian populations studied were not ethnically different [30]. Three [36-38] of five [3640] other Asian studies showed significantly higher mutant genotypes in infertile groups than fertile groups of Iranian men. Similarly, studies carried out on Korean infertile men also had contrasting results [41-43].

Moreover, in Caucasian populations, few studies reported that the polymorphism in MTHFR was a risk of male infertility [44-48]. However, other studies inferred that the mutant genotype is not such a risk [49-58]. Moreover, few studies gave contrasting conclusions for the same population $[47,48]$.

Therefore, the differences in the results could be attributed to other factors such as variations in recruitment of subjects, sample size, ethnicity and geographic factors (Tables 3 and 4). The results are also dependent on the reproductive health (testicular, hormonal, and epididymal, etc.), general health (infections, surgeries, fever and antibodies, etc.) and lifestyle factors (clothing, occupation, smoking, caffeine intake, etc.), which were not addressed while selecting subjects in many of the previous studies. There were eight studies [18,22,23,26,27,31,37,49] that showed association of $677 \mathrm{~T}$ with male infertility and seven studies $[20,39,40,50-52,58]$ that lack association and did not mention criteria of selection of infertile men. Furthermore, nine studies [23,24,26$28,32,37,38,47]$ showing association and eight studies $[19,28,34,39,40,48,50,57]$ that showed no association, did not mention selection criteria of fertile men as a control group (data not shown). 
Table 3. Allelic distribution of the MTHFR C677T mutation in infertile patients and controls from various other populations.

\begin{tabular}{|c|c|c|c|c|c|c|c|c|c|c|c|c|}
\hline \multirow{2}{*}{$\begin{array}{l}\text { Study } \\
\text { [Refs.] }\end{array}$} & \multirow[t]{2}{*}{ Country } & \multirow{2}{*}{\begin{tabular}{|c} 
Patients \\
$n$
\end{tabular}} & \multirow{2}{*}{\begin{tabular}{|c|} 
Controls \\
$n$
\end{tabular}} & \multicolumn{2}{|c|}{ Patients } & \multicolumn{2}{|c|}{ Controls } & \multirow{2}{*}{ OR } & \multirow{2}{*}{ SE } & \multirow{2}{*}{$\begin{array}{l}\text { Lower } \\
\text { Limit }\end{array}$} & \multirow{2}{*}{$\begin{array}{l}\text { Upper } \\
\text { Limit }\end{array}$} & \multirow{2}{*}{$\begin{array}{c}p \\
\text { Value }\end{array}$} \\
\hline & & & & $\mathrm{C}$ & $\mathrm{T}(\%)$ & $\mathrm{C}$ & $\mathrm{T}(\%)$ & & & & & \\
\hline \multicolumn{13}{|c|}{ African } \\
\hline [18] & Egypt & 139 & 90 & 133 & $125(48.45)$ & 126 & $54(30.00)$ & 2.19 & 0.20 & 1.47 & 3.28 & $0.000^{\mathrm{a}}$ \\
\hline [19] & Egypt & 107 & 107 & 163 & $51(23.83)$ & 156 & $58(27.10)$ & 0.84 & 0.22 & 0.54 & 1.30 & 0.438 \\
\hline$[20]$ & Algeria & 74 & 84 & 95 & $55(36.67)$ & 110 & $58(35.52)$ & 1.10 & 0.23 & 0.69 & 1.74 & 0.690 \\
\hline [21] & Morocco & 344 & 690 & 523 & $165(23.98)$ & 988 & $392(28.41)$ & 0.80 & 0.11 & 0.64 & 0.98 & 0.033 \\
\hline \multicolumn{13}{|l|}{ Asian } \\
\hline$[22]$ & China & 182 & 53 & 140 & $224(61.54)$ & 58 & $48(45.28)$ & 1.93 & 0.22 & 1.25 & 2.99 & $0.003^{\mathrm{a}}$ \\
\hline [23] & China & 355 & 252 & 420 & $290(40.85)$ & 351 & $153(30.36)$ & 1.58 & 0.12 & 1.24 & 2.02 & $0.000^{\mathrm{a}}$ \\
\hline [24] & China & 75 & 72 & 92 & $58(38.67)$ & 108 & $36(25.00)$ & 1.89 & 0.26 & 1.15 & 3.12 & $0.013^{\mathrm{a}}$ \\
\hline [25] & China & 82 & 133 & 64 & $100(60.98)$ & 133 & $133(50.00)$ & 1.56 & 0.20 & 1.05 & 2.32 & $0.027^{\mathrm{a}}$ \\
\hline [26] & China & 131 & 293 & 125 & $139(52.65)$ & 338 & $248(42.32)$ & 1.52 & 0.15 & 1.13 & 2.03 & $0.005^{\mathrm{a}}$ \\
\hline [27] & China & 290 & 90 & 216 & $364(62.76)$ & 95 & $85(47.22)$ & 1.88 & 0.17 & 1.34 & 2.64 & $0.000^{\mathrm{a}}$ \\
\hline [28] & China & 271 & 180 & 262 & $280(51.66)$ & 149 & $211(58.61)$ & 0.75 & 0.14 & 0.58 & 0.99 & $0.040^{\mathrm{a}}$ \\
\hline [29] & India & 151 & 200 & 250 & $52(17.22)$ & 363 & $37(9.25)$ & 2.04 & 0.23 & 1.30 & 3.20 & $0.002^{\mathrm{a}}$ \\
\hline$[30]$ & India & 522 & 315 & 872 & $172(16.48)$ & 560 & 70 (11.11) & 1.58 & 0.15 & 1.17 & 2.12 & $0.003^{\mathrm{a}}$ \\
\hline [31] & India & 12 & 20 & 13 & $11(45.83)$ & 37 & $3(7.50)$ & 10.44 & 0.73 & 2.51 & 43.37 & $0.001^{\mathrm{a}}$ \\
\hline [32] & India & 637 & 364 & 1048 & $226(17.74)$ & 627 & 99 (13.64) & 1.37 & 0.13 & 1.06 & 1.76 & $0.017^{\mathrm{a}}$ \\
\hline [33] & India & 206 & 230 & 358 & $54(13.11)$ & 418 & $42(9.13)$ & 1.50 & 0.22 & 0.98 & 2.30 & 0.062 \\
\hline [34] & India & 179 & 200 & 239 & $119(33.24)$ & 240 & $160(40.00)$ & 0.75 & 0.15 & 0.55 & 1.01 & 0.054 \\
\hline [35] & India & 100 & 100 & 186 & $14(7.00)$ & 181 & $19(9.50)$ & 0.72 & 0.37 & 0.35 & 1.47 & 0.365 \\
\hline [36] & Iran & 164 & 328 & 196 & $132(40.24)$ & 436 & $220(33.54)$ & 1.33 & 0.14 & 1.01 & 1.67 & $0.039^{\mathrm{a}}$ \\
\hline [37] & Iran & 118 & 132 & 161 & $75(31.78)$ & 206 & $58(21.97)$ & 1.65 & 0.20 & 1.11 & 2.47 & $0.014^{\mathrm{a}}$ \\
\hline$[38]$ & Iran & 242 & 255 & 327 & $157(32.44)$ & 386 & $124(24.31)$ & 1.49 & 0.14 & 1.13 & 1.97 & $0.005^{\mathrm{a}}$ \\
\hline [39] & Iran & 266 & 77 & 402 & $130(24.44)$ & 74 & $80(51.95)$ & 0.30 & 0.19 & 0.21 & 0.43 & 0.100 \\
\hline [40] & Iran & 300 & 303 & 430 & $170(28.33)$ & 426 & $180(29.70)$ & 0.94 & 0.13 & 0.73 & 1.20 & 0.600 \\
\hline [41] & Korea & 371 & 396 & 410 & $332(44.73)$ & 490 & $302(38.13)$ & 1.31 & 0.10 & 1.07 & 1.61 & $0.009^{\mathrm{a}}$ \\
\hline [42] & Korea & 360 & 325 & 411 & $309(42.92)$ & 402 & $248(38.15)$ & 1.22 & 0.11 & 0.98 & 1.51 & 0.073 \\
\hline$[43]$ & Korea & 85 & 246 & 104 & $66(38.82)$ & 280 & $212(43.09$ & 0.84 & 0.18 & 0.59 & 1.20 & 0.332 \\
\hline \multicolumn{13}{|c|}{ Caucasian } \\
\hline [44] & Brazil & 156 & 233 & 222 & $90(28.85)$ & 387 & $79(16.95)$ & 1.99 & 0.18 & 1.41 & 2.80 & $0.000^{\mathrm{a}}$ \\
\hline [45] & Brazil & 133 & 173 & 183 & $83(68.80)$ & 299 & $47(13.85)$ & 2.89 & 0.21 & 1.93 & 4.31 & $0.000^{\mathrm{a}}$ \\
\hline [46] & Slovenia & 100 & 111 & 109 & $91(45.50)$ & 144 & $78(35.14)$ & 1.54 & 0.20 & 1.04 & 2.28 & $0.030^{\mathrm{a}}$ \\
\hline [47] & Italy & 59 & 46 & 54 & $64(54.24)$ & 57 & $35(38.04)$ & 1.93 & 0.28 & 1.11 & 3.36 & $0.020^{\mathrm{a}}$ \\
\hline [48] & Italy & 93 & 105 & 111 & $75(40.32)$ & 119 & $91(43.33)$ & 0.88 & 0.20 & 0.59 & 1.32 & 0.545 \\
\hline [49] & Turkey & 100 & 50 & 132 & $68(34.00)$ & 80 & $20(20.00)$ & 2.06 & 0.29 & 1.16 & 3.65 & $0.013^{\mathrm{a}}$ \\
\hline$[50]$ & Germany & 255 & 200 & 321 & $189(37.09)$ & 273 & $127(31.75)$ & 1.27 & 0.14 & 0.96 & 1.67 & 0.095 \\
\hline [51] & The Netherlands & 77 & 113 & 112 & $42(27.27)$ & 148 & $78(34.51)$ & 0.71 & 0.23 & 0.45 & 1.11 & 0.137 \\
\hline$[52]$ & Serbia & 52 & 56 & 68 & $36(34.62)$ & 72 & $40(35.71)$ & 0.95 & 0.29 & 0.54 & 1.67 & 0.866 \\
\hline [53] & Poland & 284 & 352 & 399 & $169(29.75)$ & 482 & $222(31.53)$ & 0.92 & 0.12 & 0.72 & 1.17 & 0.494 \\
\hline [54] & Jordan & 150 & 150 & 197 & $103(34.33)$ & 215 & $85(28.33)$ & 1.32 & 0.18 & 0.97 & 1.87 & 0.114 \\
\hline [55] & Sweden & 149 & 182 & 209 & 89 (29.87) & 261 & $103(28.30)$ & 1.08 & 0.17 & 0.77 & 1.51 & 0.658 \\
\hline$[56]$ & France & 250 & 114 & 337 & $163(32.00)$ & 150 & $78(34.21)$ & 0.93 & 0.17 & 0.67 & 1.30 & 0.668 \\
\hline [57] & Russia & 180 & 301 & 250 & $110(30.56)$ & 421 & $181(30.07)$ & 1.02 & 0.14 & 0.77 & 1.36 & 0.873 \\
\hline$[58]$ & Canada & 39 & 19 & 58 & $20(51.22)$ & 21 & $17(44.74)$ & 0.43 & 0.42 & 0.19 & 0.96 & $0.041^{\mathrm{a}}$ \\
\hline
\end{tabular}

OR: odds ratio; SE: standard error.

${ }^{\text {a } A} p$ value of $<0.05$ was considered to be significant. 
BALKAN JOURNAL OF MEDICAL GENETICS

Irfan M, Ismail M, Azhar Beg M, Shabbir A, Rashid Kayani A, Kaukab Raja G

Table 4. Genetic distribution of the MTHFR C677T mutation in infertile patients and controls from various other populations.

\begin{tabular}{|c|c|c|c|c|c|c|c|c|c|c|c|c|}
\hline \multirow{2}{*}{ 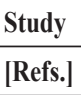 } & \multirow[t]{2}{*}{ Country } & \multicolumn{2}{|c|}{ Patients Controls } & \multicolumn{2}{|c|}{ Patients } & \multicolumn{2}{|c|}{ Controls } & \multirow{2}{*}{ OR } & \multirow{2}{*}{ SE } & \multirow{2}{*}{$\begin{array}{c}\text { Lower } \\
\text { Limit }\end{array}$} & \multirow{2}{*}{$\begin{array}{l}\text { Upper } \\
\text { Limit }\end{array}$} & \multirow{2}{*}{$\begin{array}{c}p \\
\text { Value }\end{array}$} \\
\hline & & $n$ & $n$ & $\mathrm{CC}$ & CT + TT (\%) & $\mathrm{CC}$ & CT + TT (\%) & & & & & \\
\hline \multicolumn{13}{|c|}{ African } \\
\hline$[18]$ & Egypt & 139 & 90 & 109 & $133(54.96)$ & 144 & $111(43.53)$ & 1.58 & 0.18 & 1.11 & 2.26 & $0.011^{2}$ \\
\hline [19] & Egypt & 107 & 107 & 55 & $84(60.43)$ & 41 & $49(54.44)$ & 1.28 & 0.27 & 0.75 & 2.18 & 0.370 \\
\hline [20] & Algeria & 74 & 84 & 31 & $43(58.11)$ & 36 & $48(57.14)$ & 1.04 & 0.32 & 0.55 & 1.96 & 0.902 \\
\hline [21] & Morocco & 344 & 690 & 199 & $145(42.15)$ & 351 & $339(49.13)$ & 0.75 & 0.13 & 0.58 & 0.98 & 0.034 \\
\hline \multicolumn{13}{|l|}{ Asian } \\
\hline [22] & China & 182 & 53 & 27 & $155(85.16)$ & 15 & $38(71.70)$ & 2.27 & 0.37 & 1.10 & 4.67 & 0.027 \\
\hline [23] & China & 355 & 252 & 130 & $225(63.38)$ & 128 & $124(49.21)$ & 1.79 & 0.17 & 1.29 & 2.48 & $0.001^{2}$ \\
\hline [24] & China & 75 & 72 & 27 & $48(64.00)$ & 40 & $32(44.44)$ & 2.22 & 0.34 & 1.15 & 4.31 & 0.018 \\
\hline [25] & China & 82 & 133 & 14 & $68(82.93)$ & 36 & $97(72.93)$ & 1.80 & 0.35 & 0.90 & 3.60 & 0.095 \\
\hline [26] & China & 131 & 293 & 35 & $97(73.48)$ & 98 & $293(74.94)$ & 0.93 & 0.23 & 0.59 & 1.45 & 0.741 \\
\hline [27] & China & 290 & 90 & 39 & $251(86.55)$ & 24 & $66(73.33)$ & 2.34 & 0.29 & 1.32 & 4.16 & 0.004 \\
\hline [28] & China & 271 & 180 & 75 & $196(72.32)$ & 32 & $148(82.22)$ & 0.57 & 0.24 & 0.35 & 0.90 & 0.016 \\
\hline [29] & India & 151 & 200 & 105 & $46(30.46)$ & 163 & $37(18.50)$ & 1.93 & 0.25 & 1.17 & 3.17 & $0.010^{2}$ \\
\hline [30] & India & 522 & 315 & 378 & $144(27.59)$ & 251 & $64(20.32)$ & 1.49 & 0.17 & 1.07 & 2.09 & 0.019 \\
\hline [31] & India & 12 & 20 & 4 & $8(66.67)$ & 18 & $2(10.00)$ & 18.00 & 0.96 & 2.72 & 19.23 & $0.003^{3}$ \\
\hline [32] & India & 637 & 364 & 447 & $190(29.83)$ & 275 & $89(24.45)$ & 1.31 & 0.15 & 0.98 & 1.76 & 0.068 \\
\hline [33] & India & 206 & 230 & 158 & $48(23.30)$ & 188 & $42(18.26)$ & 1.36 & 0.24 & 0.85 & 2.16 & 0.195 \\
\hline [34] & India & 179 & 200 & 81 & $98(54.75)$ & 70 & $130(65.00)$ & 0.65 & 0.21 & 0.43 & 0.99 & $0.042^{2}$ \\
\hline [35] & India & 100 & 100 & 86 & $14(14.00)$ & 81 & $19(19.00)$ & 0.69 & 0.38 & 0.33 & 1.48 & 0.342 \\
\hline [36] & Iran & 164 & 328 & 58 & $106(64.63)$ & 144 & $184(56.10)$ & 1.43 & 0.20 & 0.97 & 2.11 & 0.070 \\
\hline [37] & Iran & 118 & 132 & 51 & $67(56.78)$ & 77 & $55(41.67)$ & 1.84 & 0.26 & 1.11 & 3.04 & 0.017 \\
\hline [38] & Iran & 242 & 255 & 109 & $133(54.96)$ & 144 & $111(43.53)$ & 1.58 & 0.18 & 1.11 & 2.26 & $0.011^{2}$ \\
\hline [39] & Iran & 266 & 77 & 153 & $113(42.48)$ & 33 & $44(57.14)$ & 0.55 & 0.26 & 0.33 & 0.92 & 0.024 \\
\hline [40] & Iran & 300 & 303 & 161 & $139(46.33)$ & 151 & $152(50.17)$ & 0.86 & 0.16 & 0.62 & 1.18 & 0.347 \\
\hline [41] & Korea & 371 & 396 & 103 & $268(72.24)$ & 145 & $251(63.38)$ & 1.50 & 0.16 & 1.11 & 2.04 & $0.009^{\prime}$ \\
\hline [42] & Korea & 360 & 325 & 115 & $245(68.06)$ & 118 & $207(63.69)$ & 1.21 & 0.16 & 0.88 & 1.67 & 0.229 \\
\hline [43] & Korea & 85 & 246 & 30 & $55(64.71)$ & 87 & $159(64.63)$ & 1.00 & 0.26 & 0.60 & 1.68 & 0.990 \\
\hline \multicolumn{13}{|c|}{ Caucasian } \\
\hline [44] & Brazil & 156 & 233 & 81 & $75(48.08)$ & 167 & $66(28.33)$ & 2.34 & 0.22 & 1.53 & 3.58 & $0.000^{\circ}$ \\
\hline [45] & Brazil & 133 & 173 & 66 & $67(50.38)$ & 136 & $37(21.39)$ & 3.73 & 0.25 & 2.27 & 6.14 & $0.000^{2}$ \\
\hline [46] & Slovenia & 100 & 111 & 29 & $71(71.00)$ & 47 & $64(57.66)$ & 1.80 & 0.29 & 1.01 & 3.19 & $0.045^{\prime}$ \\
\hline [47] & Italy & 59 & 46 & 11 & $48(81.36)$ & 18 & $28(60.87)$ & 2.81 & 0.45 & 1.16 & 6.78 & 0.022 \\
\hline [48] & Italy & 93 & 105 & 37 & $56(60.22)$ & 43 & $62(59.05)$ & 1.05 & 0.29 & 0.59 & 1.85 & 0.867 \\
\hline [49] & Turkey & 100 & 50 & 44 & $56(56.00)$ & 30 & $20(40.00)$ & 1.91 & 0.35 & 0.96 & 3.81 & 0.066 \\
\hline [50] & Germany & 255 & 200 & 114 & $141(55.29)$ & 92 & $108(54.00)$ & 1.05 & 0.19 & 0.73 & 1.53 & 0.783 \\
\hline [51] & The Netherlands & 77 & 113 & 42 & $35(45.45)$ & 50 & $63(55.75)$ & 0.66 & 0.30 & 0.37 & 1.18 & 0.164 \\
\hline$[52]$ & Serbia & 52 & 56 & 22 & $30(57.69)$ & 23 & $33(58.93)$ & 0.95 & 0.39 & 0.44 & 2.04 & 0.896 \\
\hline [53] & Poland & 284 & 352 & 143 & $256(64.16)$ & 166 & $316(65.56)$ & 0.94 & 0.14 & 0.71 & 1.24 & 0.665 \\
\hline [54] & Jordan & 150 & 150 & 67 & $83(55.33)$ & 74 & $76(50.67)$ & 1.21 & 0.23 & 0.77 & 1.90 & 0.418 \\
\hline [55] & Sweden & 149 & 182 & 73 & $76(51.01)$ & 94 & $88(48.35)$ & 1.11 & 0.22 & 0.72 & 1.72 & 0.631 \\
\hline$[56]$ & France & 250 & 114 & 118 & $132(52.80)$ & 49 & $65(57.02)$ & 0.84 & 0.23 & 0.54 & 1.32 & 0.454 \\
\hline [57] & Russia & 180 & 301 & 89 & $91(50.56)$ & 153 & $148(49.17)$ & 1.06 & 0.19 & 0.73 & 1.53 & 0.769 \\
\hline [58] & Canada & 39 & 19 & 22 & $17(43.59)$ & 8 & $11(57.89)$ & 0.56 & 0.57 & 0.19 & 1.70 & 0.308 \\
\hline
\end{tabular}

OR: odds ratio; SE: standard error.

a A $p$ value of $<0.05$ was considered to be significant. 
In addition, the MTHFR C677T association with male infertility was also affected by folate supplementation, usually prescribed in infertility. The status of folate and supplementation is not mentioned in the previous studies except four $[34,36,42,55]$.

Although a single study that took folate levels as a factor of infertility in Caucasian men also found no association of $677 \mathrm{~T}$ with male infertility, we can assume, depending on the socioeconomic and health facilities that the Caucasians may have comparatively more chances of folate supplementation than Asians, thus lowering the association of $677 \mathrm{~T}$ with infertility $[42,59,60]$. The previous studies described that $35.0 \%$ of the Pakistani population is folate deficient (61) and no organized folate supplementation program is currently working for the general population.

\section{CONCLUSIONS}

The present study of the association between idiopathic sperm disorders and the MTHFR 677T polymorphism suggests that the polymorphism might be a factor of male infertility in Pakistan. The results showed a significantly $(p<0.05)$ higher T allele and gene frequencies in the infertile men as compared to the fertile men. Furthermore, the carriers of the $\mathrm{T}$ allele were more in all semen groups of infertile men as compared to the control group. Association of male infertility with MTHFR C677T polymorphisms provokes investigation of the effects of folic acid supplementation in infertile men on sperm parameters. However, the present study is about only one ethnic group in Pakistan, which was self-defined by the subjects, while, MTHFR 677T frequencies in the Pakistani populations have been reported to be greatly variable and may influence the frequencies of SNPs. Therefore, studies must be conducted to investigate the association of SNPs also at the ethnic level. Secondly, the screening of infertile men must include karyotyping that was not done in the present study. We suggest that further studies must consider folate levels and folate supplementation while selecting subjects in genetic studies. Similarly, the studies must consider other well defined factors of reproductive health, general health and lifestyle factors in exclusion criteria. Further research, analyzing multiple fertility regions/loci in idiopathic infertile men is also required through high throughput analyzers, i.e., microarray, etc.
Declaration of Interest. The authors report no conflicts of interest. The authors alone are responsible for the content and writing of this article.

\section{REFERENCES}

1. WHO. Manual for the standardised investigation and diagnosis of the infertile couple. World Health Organisation. Cambridge, United Kingdom: Cambridge University Press. 2000: 79-82.

2. Tahir F, Shahab M, Afzal M, Subhan F, Sultan S, Kazi BM, et al. Male reproductive health: An important segment towards improving reproductive health of a couple. In: Chaudhry MA, Desousa M, Eds. Population Research and Policy Development in Pakistan. Islamabad, Pakistan: Population Association of Pakistan. 2004: 227248.

3. Egoczue S, Blanco J, Vendrell JM. Human male infertility: Chromosome anomalies, meiotic disorders, abnormal spermatozoa and recurrent abortion. Hum Reprod Update. 20006; 6(1): 93 105.

4. Hargreave T. Genetically determined male infertility and assisted reproduction techniques. J Endocrinol Invest. 2000; 23(10): 697-710.

5. Boxmeer JC, Smit M, Utomo E, Romijn JC, Eijkemans MJ, Lindemans J, et al. Low folate in seminal plasma is associated with increased sperm DNA damage. Fertil Steril. 2009; 92(2): 548-556.

6. Donnelly JG. Folic acid. Crit Rev Clin Lab Sci. 2001; 38(3): 183-223.

7. Fowler B. Homocysteine: Overview of biochemistry, molecular biology, and role in disease processes. Semin Vasc Med. 2005; 5(2): 77-86.

8. Goyette P, Pai A, Milos R, Frosst P, Tran P, Chen $Z$, et al. Gene structure of human and mouse methylenetetrahydrofolate reductase (MTHFR). Mamm Genome. 1998; 9(8): 652-656.

9. Li E. Chromatin modification and epigenetic reprogramming in mammalian development. Nat Rev Genet. 2002; 3(9): 662-673.

10. Gaughan DJ, Kluijtmans LA, Barbaux S, McMaster D, Young IS, Yarnell JW, et al. The methionine synthase reductase (MTRR) A66G polymorphism is a novel genetic determinant of 
plasma homocysteine concentrations. Atherosclerosis. 2001; 157(2): 451-456.

11. Frosst $\mathrm{P}, \mathrm{Blom}$ HJ, Milos R, Goyette P, Sheppard CA, Matthews RG, et al. A candidate genetic risk factor for vascular disease: A common mutation in methylene-tetrahydrofolate reductase. Nat Genet. 1995; 10(1): 111-113.

12. Eaton WW, Smith C, Ybarra M, Muntaner C, Tien A. Centre for epidemiological studies depression scale: Review and revision (CESD and CESD-R). In: Maruish ME, Ed. The Use of Psychological Testing for Treatment, Panning and Outcome Assessment, 3rd ed. (Volume 3: Instruments for Adults.) Mahwah, NJ, USA: Lawrence Erlbaum. 2004: 363-377.

13. Hamilton M. The assessment of anxiety states by rating. Br J Med Psychol. 1959; 32(1): 50-55.

14. Holmes TH, Rahe TH. The social readjustment rating scale. J Psychomatic Res. 1967; 11(2): 213-221.

15. Jacques PF, Bostom AG, Williams RR, Ellison $\mathrm{RC}$, Eckfeldt JH, Rosenberg IH, et al. Relation between folate status, a common mutation in methylenetetrahydrofolate reductase, and plasma homocysteine concentrations. Circulation. 1996; 93(1): 7-9.

16. Sheweita SA, Tilmisany AM, Al-Sawaf H. Mechanisms of male infertility: Role of antioxidants. Curr Drug Metab. 2005; 6(5): 495-501.

17. Klein R, Pfitzer P. Flow cytometry of postmortem human testicular tissue in cases of atherosclerosis. Cytometry. 1984; 5(6): 636-643.

18. Zalata AA, Badawy A, Mokhtar N, Serria MS, Abdeen HM, Alghobary M, et al. Mutations in the methy-lenetetrahydrofolate reductase gene and methionine metabolism in male infertility. Bull Egypt Soc Physiol Sci. 2011; 31(2): 221-240.

19. Hussein TM, Elneely DI. Y-chromosome microdeletions and the MTHFR C677T polymorphism in Egyptian men with nonobstructive azoospermia. Hum Androl. 2014; 4(3): 66-70.

20. Chellat D, Rezgoune ML, Hamane D, Semmame $\mathrm{O}$, Benlatréche C, Abadi N, et al. Genet Test Mol Bio-markers. 2012; 16(8): 874-878.

21. Eloualid A, Abidi O, Charif M, El Houate B, Benrahma H, Louanjli N, et al. Association of the MTHFR A1298C variant with unexplained severe male infertility. PLoS One. 2012; 7(3): e34111. doi: 10.1371/journal.pone. 0034111.

22. Sun HT, Zhang JY, Lu YJ. Associated of the meth-ylenetetrahydrofolate reductase gene C677T polymorphism with male infertility. Reprod Contracept. 2007; 27(7): 443-446.

23. A ZC, Yang Y, Zhang SZ, Li N, Zhang W. Single nucleotide polymorphism C677T in the methylenetetrahydrofolate reductase gene might be a genetic risk factor for infertility for Chinese men with azoospermia or severe oligozoospermia. Asian J Androl. 2007; 9(1): 57-62.

24. Ling L, Zhiming C, Huimin L, Weiping Q. Association of MTHFR C677T and MS A2756G polymorphism with semen quality. J Cent South Univ. 2012; 37(10): 1054-1059.

25. Li E. Chromatin modification and epigenetic reprogramming in mammalian development. Nat Rev Genet. 2002; 3(9): 662-673.

26. Yang BH, Peng GF, Pi JP, Association of methylenetetrahydrofolate reductase gene C677T polymorphism with asthenospermia in Han population of South Anhui. Acta Acad Med Wannan. 2010; 29(1): 5-7.

27. Pei J. Association between MTHFR C677T polymorphism and male infertility in Han population of He Nan China. China Hlth Care Nutr. 2013; 2(7): 629-630.

28. Qiu XF, Hu XP, Li YJ, Dang J, Peng L, Xu X, et al. Association between methyleneterahydrofolate reductase gene C677T polymorphism and male infertility with azoospermia or severe oligozoospermia and asthenospermia in Ningxia Han population. J Ningxia Med Univ. 2011; 33(7): 625-628.

29. Singh K, Singh SK, Sah R, Singh I, Raman R. Mutation C677T in the methylenetetrahydrofolate reductase gene is associated with male infertility in an Indian population. Int J Androl. 2005; 28(2): 115-119.

30. Gupta N, Gupta S, Dama M, David A, Khanna G, Khanna A, et al. Association of $677 \mathrm{C}>\mathrm{T}$ substitution in the MTHFR gene with male infertility - A study on an Indian population and a meta-analysis. PLoS One. 2011; 6(7): e22277. doi: 10.1371/journal.pone.0022277.

31. Kumari R. Genetic interaction between methylene-tetrahydrofolate reductase C677T gene 
polymorphisms and stem cell associated "risk factor" in male infertility. Sch J App Med Sci. 2013; 1(5): 552-556

32. Naqvi H, Hussain SR, Ahmad MK, Mahdi F, Jaiswar SP, Shankhwar SN, et al. Role of $677 \mathrm{C} \rightarrow$ T polymorphism a single substitution in methylenetetrahydro-folate reductase (MTHFR) gene in North Indian infertile men. Mol Biol Rep. 2014; 41(2): 573-579.

33. Vani GT, Mukesh N, Devi PR, Rani PU, Reddy PP. Methylenetetrahydrofolate reductase C677T polymorphism is not associated with male infertility in a South Indian population. Andrologia. 2012; 44 (1): 252-259.

34. Dhillon VS, Shahid M, Husain SA. Associations of MTHFR DNMT3b 4977bp deletion in mtDNA and GSTM1 deletion, and aberrant $\mathrm{CpG}$ island hypermethylation of GSTM1 in nonobstructive Infertility in Indian men. Mol Hum Reprod. 2007; 13(4): 213-222.

35. Kumar K, Venkatesh S, Sharma PR, Tiwari PK, Dada R. DAZL 260A $>\mathrm{G}$ and MTHFR $677 \mathrm{C}>\mathrm{T}$ variants in sperm DNA of infertile Indian men. Indian J Biochem Biophys. 2011; 48(6): 422-426.

36. Safarinejad MR, Shafiei N, Safarinejad S. Relationship between genetic polymorphisms of methylene-tetrahydrofolate reductase (C677T, A1298C, and G1793A) as risk factors for idiopathic male infertility. Reprod Sci. 2011; 18(3): 304-315.

37. Karimian M, Colagar AH. Association of C677T transition of the human methylenetetrahydrofolate reductase (MTHFR) gene with male infertility. Reprod Fertil Dev. 2016; 28(6): 785-794. doi: 10.1071/RD14186.

38. Nikzad H, Karimian M, Sareban K, Khoshsokhan M, Colagar AH. MTHFR-Ala222Val and male infertility: A study in Iranian men, an updated meta-analysis and an in silico-analysis. Reprod BioMed Online. 2015; 31(5): 668-680.

39. Rayegani M, Sadeghi MR, Yaghmaei B, Akhoundi MA, Lakpour N, Hojat M, et al. Comparison of C677T polymorphism of the methylenetetrahydrofolate reductase (MTHFR) gene in oligo-asthenoteratozoospermic (OAT) and normozoospermic iranian men. (Abstract O-51). Abstracts of the 15th Congress of the Iranian Society for Reproductive Medicine, held on 18-
20 February 2009 at Tehran, Iran. Iran J Reprod Med. 2009; 7(Suppl 1): 24.

40. Bitarafan F, Noruzinia M, Salehi Z, Rahaee S, Sadeghiani G, Mohammadi-Fateh S. Methylenetetra-hydrofolate reductase (MTHFR) C677T polymorphism and male infertility in an Iranian population group. (Abstract P-130) Proceedings of the 3rd International and 18th National Congress of the Iranian Society for Reproductive Medicine, held on 18-20 April, 2012, at Tabriz, Iran. Tabriz, Iran: Iranian Society for Reproductive Medicine. 2012: 87-88.

41. Park JH, Lee HC, Jeong YM, Chung TG, Kim HJ, Kim NM, et al. MTHFR C677T polymorphism associates with unexplained infertile male factors. J Assist Reprod Genet. 2005; 22(9-10): 361-368.

42. Lee HC, Jeong YM, Lee SH, Cha KY, Song $\mathrm{SH}$, Kim NK, et al. Association study of four polymorphisms in three folate-related enzyme genes with non-obstructive male infertility. Hum Reprod. 2006; 21(12): 3162-3170.

43. Kim SY, Lim JW, Kim JW, Park SY, Seo JT. Association between genetic polymorphisms in folate-related enzyme genes and infertile men with non-obstructive azoospermia. Syst Biol Reprod Med. 2015; 61(5): 286-292.

44. Gava MM, Chagas Ede O, Bianco B, Christofolini DM, Pompeo AC, Glina S, et al. Methylenetetrahydrofolate reductase polymorphisms are related to male infertility in Brazilian men. Genet Test Mol Biomarkers. 2011; 15(3): 153-157.

45. Gava MM, Kayaki EA, Bianco B, Teles JS, Christofolini DM, Pompeo AC, et al. Polymorphisms in folate-related enzyme genes in idiopathic infertile Brazilian men. Reprod Sci. 2011; 18(12): 1267-1272.

46. Stangler Herodež Š, Zagradišnik B, Erjavec Škerget A, Zagorac A, Takač I, Vlaisavljevič V, et al. MTHFR C677T and A1298C genotypes and haplotypes in Slovenian couples with unexplained infertility problems and in embryonic tissues from spontaneous abortions. Balkan $\mathrm{J}$ Med Genet. 2013; 16(1): 31-39.

47. Paracchini V, Garte S, Taioli E. MTHFR C677T polymorphism, GSTM1 deletion and male infertility: A possible suggestion of a gene-gene interaction? Biomarkers. 2006; 11(1): 53-60. 
48. Stuppia L, Gatta V, Scarciolla O, Colosimo A, Guanciali-Franchi P, Calabrese G, et al. The methylenetetrahydrofolate reductase (MTHFR) C677T polymorphism and male infertility in Italy. J Endocrinol Invest. 2003; 26(7): 620-622.

49. Tetik A, Aliyeva U, Cetintas VB, Semerci B, Topcuoglu N. Influence of methylenetetrahydrofolate reductase (MTHFR) 677C $>$ T and $1298 \mathrm{~A}>\mathrm{C}$ gene poly-morphisms on male infertility in turkish infertile men with azoospermia and oligozoospermia. Eur Urol Suppl. 2008; 7(3): 92. doi: 10.1016/S1569-9056(08)60088-3.

50. Bezold G, Lange M, Peter RU. Homozygous meth-ylenetetrahydrofolate reductase C677T mutation and male infertility. N Engl J Med. 2001; 344(15): 1172-1173.

51. Ebisch IM, van Heerde WL, Thomas CM, van der Put N, Wong WY, Steegers-Theunissen RP. C677T Meth-ylenetetrahydrofolate reductase polymorphism interferes with the effects of folic acid and zinc sulfate on sperm concentration. Fertil Steril. 2003; 80(5): 1190-1194

52. Dordevic V, Ljujic M, Nestorovic A, Ristanovic M, Radojkovic D. Combined effect of GSTM1 gene deletion, GSTT1 gene deletion and MTHFR C677T mutation in male infertility. Arch Biol Sci Belgrade. 2010; 63(3): 525-530.

53. Kurzawski M, Wajda A, Malinowski D, Kazienko A, Kurzawa R, Drozdzik M. Association study of folate-related enzymes (MTHFR, MTR, MTRR) genetic variants with non-obstructive male infertility in a Polish population. Genet Mol Biol. 2015; 38(1): 42-47.

54. Mfady DS, Sadiq MF, Khabour OF, Fararjeh AS, Abu-Awad A, Khader Y. Associations of variants in MTHFR and MTRR genes with male infertility in the Jordanian population. Gene. 2014; 536(1): 40-44.
55. Murphy LE, Mills JL, Molloy A, Qian C, Carter $\mathrm{TC}$, Strevens $\mathrm{H}$, et al. Folate and vitamin B12 in idiopathic male infertility. Asian J Androl. 2011; 13(6): 856-861.

56. Ravel C, Chantot-Bastaraud S, Chalmey C, Barreiro L, Aknin-Seifer I, Pfeffer J, et al. Lack of association between genetic polymorphisms in enzymes associated with folate metabolism and unexplained reduced sperm counts. PLoS One. 2009; 4(8): e6540. doi: 10.1371/ journal. pone. 0006540 .

57. Weiner AS, Boyarskikh UA, Voronina EN, Tupikin AE, Korolkova OV, Morozov IV, et al. Polymorphisms in folate-metabolizing genes and risk of idiopathic male infertility: a study on a Russian population and a meta-analysis. Fertil Steril. 2014; 101(1): 87-94.

58. Ng R, Louie K, Poon K, Chow V, Ma S. Association of single nucleotide polymorphisms (SNPs) in methylenetetrahydrofolate reductase (mthfr) and male infertility. Fertil Steril. 2014; 102(3): e192. http://dx.doi.org/ 10.1016/j.fertnstert.2014.07.648.

59. Parodi PW. The French paradox unmasked: The role of folate. Med Hypotheses 1997; 49(4): 313-318.

60. Pelucchi C, Mereghetti M, Talamini R, Negri E, Montella M, Ramazzotti V, et al. Dietary folate, alcohol consumption, and risk of ovarian cancer in an Italian case-control study. Cancer Epidemiol Biomarkers Prev. 2005; 14(8): 2056-2058.

61. Iqbal MP, Lindblad BS, Mehboobali N, Yusuf FA, Khan AH, Iqbal SP. Folic acid and vitamin B6 deficiencies related hyperhomocysteinemia in apparently healthy Pakistani adults; is mass micronutrient supplementation indicated in this population? J Coll Physicians Surg Pak. 2009; 19(5): 308-312. 
